\title{
Clinical significance and functional role of transmembrane protein 47 (TMEM47) in chemoresistance of hepatocellular carcinoma
}

\author{
KEVIN TAK-PAN NG ${ }^{1,2}$, OSCAR WAI-HO YEUNG ${ }^{1}$, JIANG LIU ${ }^{1}$, CHANG XIAN LI ${ }^{1}$, \\ HUI LIU ${ }^{1}$, XIAO BING LIU ${ }^{1}$, XIANG QI $^{1}$, YUEN YUEN MA ${ }^{1}$, YIN FAN LAM ${ }^{1}$, MATTHEW YH LAU ${ }^{1}$, \\ WEN QI QIU ${ }^{1}$, HOI CHUNG SHIU ${ }^{1}$, MAN KIT LAI ${ }^{1}$, CHUNG MAU LO $^{1}$ and KWAN MAN ${ }^{1}$ \\ ${ }^{1}$ Department of Surgery, The University of Hong Kong Shenzhen Hospital and LKS Faculty of Medicine, \\ The University of Hong Kong, Hong Kong, SAR; ${ }^{2}$ The University of Hong Kong Shenzhen Institute of \\ Research and Innovation, Shenzhen, Guangdong 518057, P.R. China
}

Received February 19, 2020; Accepted July 13, 2020

DOI: 10.3892/ijo.2020.5104

\begin{abstract}
Chemoresistance is the main cause of chemotherapy failure in patients with hepatocellular carcinoma (HCC). The gene encoding transmembrane protein 47 (TMEM47) was previously identified to be significantly upregulated in HCC cell lines with acquired chemoresistance. The aim of the present study was to characterize the clinical significance and function of TMEM47 in HCC chemoresistance. The results demonstrated that the TMEM47 expression levels in the tumors of patients not responding to cisplatin-based transarterial chemoembolization (TACE) treatment was significantly higher compared with those in patients who responded to TACE treatment. Moreover, analyses from clinical samples and HCC cell lines indicated that TMEM47 expression may be upregulated in HCC in response to cisplatin treatment. Furthermore, the TMEM47 mRNA expression levels were positively correlated with the degree of cisplatin resistance of HCC cells. Overexpression of TMEM47 in HCC cells significantly promoted cisplatin resistance. The present study also demonstrated that targeted inhibition of TMEM47 could significantly reduce cisplatin resistance of cisplatin-resistant HCC cells via enhancing caspase-mediated apoptosis. In addition, targeted inhibition of TMEM47 enhanced the sensitivity of cisplatin-resistant cells to cisplatin via suppressing cisplatin-induced activation of the genes involved in drug efflux and metabolism. The present study also validated that
\end{abstract}

Correspondence to: Dr Kevin Tak-Pan Ng or Professor Kwan Man, Department of Surgery, The University of Hong Kong Shenzhen Hospital and LKS Faculty of Medicine, The University of Hong Kong, 21 Sassoon Road, Hong Kong, SAR, P.R. China

E-mail: ledodes@hku.hk

E-mail: kwanman@hku.hk

Key words: transmembrane protein 47, chemoresistance, hepatocellular carcinoma, response marker, therapeutic target
TMEM47 expression was significantly correlated with multidrug resistance-associated protein 1 in patients with HCC who received TACE treatment. In conclusion, the findings of the present study demonstrated that TMEM47 may be a useful biomarker for predicting the response to chemotherapy and a potential therapeutic target for overcoming HCC chemoresistance.

\section{Introduction}

Hepatocellular carcinoma (HCC) is the sixth most common type of cancer and the fourth leading cause of cancer-related mortality worldwide (1). Liver resection and liver transplantation are the most effective surgical treatments for patients with early HCC. However, a total of $80 \%$ of patients with HCC are diagnosed at an advanced stage, when surgical treatment is not an option due to tumor progression and presence of metastatic lesions. Regional or systemic chemotherapy is the commonly implemented treatment for patients with advanced-stage HCC. However, effective chemotherapy is challenging in patients with HCC, since HCC has been found to be resistant to several anticancer drugs (2-4). Recent advances in understanding the genetic and molecular mechanisms underlying hepatocarcinogenesis have led to the discovery of different potential molecular-targeted strategies, such as the Food and Drug Administration-approved drugs sorafenib (5) and lenvatinib (6), for improving the survival of patients with advanced HCC. However, HCC chemoresistance remains a significant obstacle (7). The development of chemoresistance in $\mathrm{HCC}$, either intrinsic or acquired, is a multifactorial process involving diverse genetic, molecular and immunological mechanisms (4). Evidence from studies conducted over the past decade indicated that HCC cannot be treated by a single drug (8). Therefore, successful therapeutic strategies should be based on combinations of novel molecular-targeted drugs against $\mathrm{HCC}$ resistance, in combination with or without conventional drugs.

Cisplatin is one of the effective drugs commonly used for the treatment of solid tumors, including $\operatorname{HCC}(9,10)$. The 
mechanism of action of cisplatin and other platinum-derived drugs in anticancer therapy mainly include increase of cellular oxidative stress and reactive oxygen species, activation of p53 signaling and apoptosis pathways and inactivation of anti-apoptotic proteins $(9,11)$. However, cancers may develop diverse mechanisms to resist cisplatin-based therapies, leading to cisplatin treatment failure $(11,12)$. Studies have identified diverse cisplatin resistance-associated targets and signaling pathways, such as various membrane transporters, repair proteins, survival and anti-apoptotic signaling pathways and microRNAs $(11,13,14)$. Due to the multifactorial nature of cisplatin resistance in cancer, the research for improving the efficacy of cisplatin-based anticancer treatment was shifted towards the development of combination therapies of cisplatin with other drugs and the reduction of cisplatin cytotoxicity $(9,11,13)$. Cisplatin is a drug commonly used in HCC for transarterial chemoembolization (TACE) therapy, which is a standard chemotherapy reported to exhibit low toxicity and improve survival for patients with intermediateor advanced-stage HCC $(10,15,16)$. The major clinical issue of cisplatin-based TACE treatment in HCC is the relatively low response rate, which is mainly due to the chemoresistant characteristics of HCC (15-17). Combination of molecular-targeted therapy with cisplatin-based TACE has been reported as a feasible strategy for overcoming treatment resistance in HCC (10).

We have previously established different chemoresistant HCC cell lines and identified several novel resistance-associated genes by cDNA microarray analysis (18). Transmembrane protein 47 (TMEM47), one of the identified candidate genes, has been found to be differentially upregulated in cisplatinand doxorubicin-resistant HCC cell lines (18). TMEM47 plays important roles in regulating the morphology and assembly rate of tight junctions from adherens junctions in vertebrates via regulating the localization of tight junction proteins (19). The roles of TMEM47 in cancer are diverse. In breast cancer, TMEM47 is one of the genes found to be overexpressed in metastatic breast cancer cells (20). Using weighted gene co-expression network analysis, TMEM47 was identified to be associated with an aggressive phenotype of breast cancer cells (20). Additionally, TMEM47 is one of the genes found to be deregulated in Ewing family tumors (21). By contrast, TMEM47 was identified as one of the tumor suppressor genes in malignant melanoma (22). To date, the role and clinical relevance of TMEM47 in HCC chemoresistance remain to be fully elucidated. The aim of the present study was to investigate the clinical relevance of TMEM47 in patients with HCC who receive chemotherapy and its functional role in HCC chemoresistance, in order to determine the prognostic and therapeutic potential of TMEM47 in patients with HCC.

\section{Materials and methods}

Patients and samples. The present study involved 27 patients with HCC who received TACE with cisplatin as the first-line treatment option (TACE group) and subsequently underwent liver resection between February 1993 and August 2010 at the Department of Surgery, University of Hong Kong Shenzhen Hospital. The technical details of the TACE protocol have been described in a previous study and cisplatin is the standard anticancer drug used in TACE treatment (23). Patients with HCC who were treated with TACE were sub-classified into two groups as follows: Response group (complete response to TACE treatment) and non-response group (without complete response to TACE treatment). A total of 30 patients with HCC who received liver resection without previous TACE treatment (non-TACE group) were enrolled from the same department. The clinicopathological parameters of the TACE and non-TACE groups are summarized in Table I. Tumor and adjacent non-tumor tissues from all patients were obtained from the Surgical Tissue Bank at the Department of Surgery of University of Hong Kong Shenzhen Hospital. All the clinical samples were collected from the patients after obtaining signed informed consent, and the collection of samples by the Surgical Tissue Bank was approved by the Ethics Committee of Institutional Review Board of the University of Hong Kong/Hospital Authority Hong Kong West Cluster (HKU/HA HKW IRB, ref. no. UW 05-359 T/1022).

Cell lines and chemicals. The human metastatic HCC cell line MHCC97L was provided by the Liver Cancer Institute at Zhongshan Hospital of Fudan University (Shanghai, China) (24). The human normal liver cell line MIHA was purchased from American Type Culture Collection (ATCC). The MHCC97L cell line was authenticated by Pangenia Lifesciences Ltd. The MHCC97L/CisR and MHCC97L/CisR2 cisplatin-resistant cell lines were established from the MHCC97L cell line by chronic incubation with increasing concentrations of cisplatin from 100 to $5,000 \mathrm{ng} / \mathrm{ml}$ for 12 months (18). The HCC cell lines were cultured in DMEM high-glucose (Gibco; Thermo Fisher Scientific, Inc.) supplemented with 10\% FBS (Gibco; Thermo Fisher Scientific, Inc.), $1 \%$ penicillin and streptomycin in a $37^{\circ} \mathrm{C}$ incubator with $5 \% \mathrm{CO}_{2}$. Cisplatin was purchased from Pharmachemie BV.

Cloning and transduction. The lentiviral open reading frame (ORF) clone of monomeric green fluorescent protein (GFP)-tagged human TMEM47 (cat. no. RC227689L2V) and lentiviral ORF control clone were purchased from OriGene Technologies, Inc. Lentiviral particles were produced by transfection into 293 cells using the Lenti-vpak Lentiviral Packaging kit (OriGene Technologies, Inc.). Suppression of TMEM47 was conducted by TMEM47-specific Mission ${ }^{\circledR}$ short hairpin RNA (shRNA) lentiviral transduction particles (Sigma-Aldrich; Merck KGaA). MISSION ${ }^{\circledR}$ TurboGFP control transduction particles (Sigma-Aldrich; Merck KGaA) was used as a control. Lentiviral particles were transduced into HCC cells according to the manufacturer's instructions. The expression levels of TMEM47 mRNA in HCC clones was examined by reverse transcription-quantitative PCR (RT-qPCR) analysis.

RT- $q$ PCR analysis. Total RNA was extracted from liver tissues or cell lines using TRIzol ${ }^{\circledR}$ reagent (Invitrogen; Thermo Fisher Scientific, Inc.). RNA was reverse-transcribed to cDNA using a High-Capacity cDNA RT kit (Applied Biosystems; Thermo Fisher Scientific, Inc.). qPCR $\left(95^{\circ} \mathrm{C}, 10 \mathrm{~min} ; 40\right.$ cycles of $95^{\circ} \mathrm{C}$ for $15 \mathrm{sec}$ and $60^{\circ} \mathrm{C}$ for $1 \mathrm{~min}$ ) was performed using the Power SYBRGreen PCR master mix and quantified using the ViiA7 Real-Time PCR System (Thermo Fisher Scientific, Inc.). $18 \mathrm{~S}$ ribosomal RNA (rRNA) was used as the internal 
Table I. Clinicopathological characteristics of TACE-treated and non-TACE-treated patients with HCC.

\begin{tabular}{|c|c|c|}
\hline $\begin{array}{l}\text { Clinicopathological } \\
\text { characteristics }\end{array}$ & $\begin{array}{c}\text { Non-TACE } \\
\text { HCC patients } \\
(\mathrm{n}=30)\end{array}$ & $\begin{array}{c}\text { TACE HCC } \\
\text { patients } \\
(\mathrm{n}=27)\end{array}$ \\
\hline \multicolumn{3}{|l|}{ Sex } \\
\hline Male & 24 & 26 \\
\hline Female & 6 & 1 \\
\hline Age (years), median (range) & $56(24-81)$ & $57(36-70)$ \\
\hline \multicolumn{3}{|l|}{ pTNM stage } \\
\hline Early (I-II) & 7 & 5 \\
\hline Advanced (III-IV) & 23 & 22 \\
\hline \multicolumn{3}{|l|}{ Venous infiltration ${ }^{\mathrm{a}}$} \\
\hline Absent & 11 & 13 \\
\hline Present & 19 & 11 \\
\hline \multicolumn{3}{|l|}{ Number of tumors } \\
\hline Single & 19 & 10 \\
\hline Multiple & 11 & 17 \\
\hline \multicolumn{3}{|l|}{ Tumor size (cm) } \\
\hline$<5$ & 16 & 11 \\
\hline$\geq 5$ & 14 & 16 \\
\hline \multicolumn{3}{|l|}{$\alpha$ fetoprotein level $(\mathrm{ng} / \mathrm{ml})$} \\
\hline$<20$ & 13 & 10 \\
\hline$\geq 20$ & 17 & 17 \\
\hline \multicolumn{3}{|l|}{ Hepatitis B surface antigen } \\
\hline Negative & 5 & 5 \\
\hline Positive & 25 & 22 \\
\hline
\end{tabular}

${ }^{\mathrm{a} D a t a}$ for 3 patients were not available. TACE, transarterial chemoembolization; HCC, hepatocellular carcinoma.

control for HCC tissue samples and $\beta$-actin was used as the internal control for cell lines. The relative gene expression levels were determined based on our previous studies $(25,26)$. The following primer pairs were used for qPCR: Human TMEM47 forward, 5'-TTCCTGGTGGGTTTGATTTC-3' and reverse, 5'-GTAAAGGACCAGGCTGCAAA-3'; multidrug resistance-associated protein $1(A B C C l)$ forward, 5'-CGACTT CAAACCCAGAGAGC-3' and reverse, 5'-ACAAAAGGA TCCCCCAAAAC-3'; 18S rRNA forward, 5'-CTCTTAGCT GAGTGTCCCGC-3' and reverse, 5'-CTGATCGTCTTCGAA CCTCC-3'; and $\beta$-actin forward, 5'-CTCTTCCAGCCTTCC TTCCT-3' and reverse, 5'-AGCACTGTGTTGGCGTACAG-3'.

Western blotting. Total protein was extracted from cells by using Cell Lysis Buffer (Cell Signaling Technology, Inc.). Protein quantification was performed with the Bio-Rad Protein Assay Dye Reagent Concentrate (Bio-Rad Laboratories, Inc.). Western blotting was performed as previously described (27) and protein signals were detected using the ECL Prime Western Blotting Detection kit (GE Healthcare). Briefly, $20 \mu \mathrm{g}$ protein was loaded per lane, resolved by $12 \%$ bis-acrylamide SDS-PAGE electrophoresis, and transferred onto PVDF membranes (EMD Millipore). The membranes were blocked in 5\% non-fat milk at room temperature for $1 \mathrm{~h}$ and then incubated with antibody against GFP protein (1:1,000 dilution, cat. no. TA180076; OriGene Technologies, Inc.) at $4^{\circ} \mathrm{C}$ overnight. Antibody against human $\beta$-actin (1:10,000 dilution, cat. no. MB1501) was purchased from Merck KGaA.

MTT cytotoxicity assay. HCC cells $\left(5 \times 10^{3}\right.$ cells per well) were seeded into a 96 -well plate and incubated under normal culture conditions $\left(37^{\circ} \mathrm{C}, 5 \% \mathrm{CO}_{2}\right)$ for $24 \mathrm{~h}$. The cells were treated with a series of cisplatin concentrations $(0-5,000 \mathrm{ng} / \mathrm{ml})$ under normal culture conditions for $72 \mathrm{~h}$. The cells were then incubated with $100 \mu \mathrm{l}$ of $5 \mathrm{mg} / \mathrm{ml}$ MTT solution (Invitrogen; Thermo Fisher Scientific, Inc.) for $3 \mathrm{~h}$ at $37^{\circ} \mathrm{C}$ until crystals were formed. MTT solution was removed from each well and the crystals were dissolved in $100 \mu \mathrm{l}$ DMSO. Color intensity was measured using a microplate reader (Bio-Rad Laboratories, Inc.) at a wavelength of $570 \mathrm{~nm}$. Each experiment consisted of four replicates and at least three individual experiments were performed.

Colony formation assay. HCC cells $\left(5 \times 10^{2}\right.$ cells per well) were seeded into a 6-well plate and incubated under normal culture conditions for $24 \mathrm{~h}$. The cells were treated with a series of cisplatin concentrations for 2 weeks. Cells were fixed with ice-cold methanol at $-20^{\circ} \mathrm{C}$ for $30 \mathrm{~min}$ and stained with $0.01 \%$ $(\mathrm{w} / \mathrm{v})$ crystal violet solution at room temperature for $10 \mathrm{~min}$. The number of colonies (defined as $>50$ cells) visible to the naked eye was counted directly on the plate. Each experiment consisted of two replicates and at least three individual experiments were performed. Statistical significance was calculated from three independent experiments.

Apoptosis assay. $\mathrm{HCC}$ cells ( $3 \times 10^{5}$ cells per well) were seeded into a 6-well plate for $24 \mathrm{~h}$. The cells were treated with a series of cisplatin concentrations $(1,000-5,000 \mathrm{ng} / \mathrm{ml})$ for $72 \mathrm{~h}$. The cells were harvested and washed with PBS twice for 5 min per wash. The cells were suspended and incubated with propidium iodide (PI)-containing Annexin-V-FLUOS labeling solution (Roche Diagnostics) for $15 \mathrm{~min}$ at room temperature. Stained cells were analyzed with a flow cytometer (BD Biosciences). For each experiment, 10,000 gated events were acquired to analyze the fluorescence intensity for Annexin-V (excitation: $488 \mathrm{~nm}$, emission: $518 \mathrm{~nm}$ ) and PI (excitation: 488-540 nm, emission: $617 \mathrm{~nm}$ ). Early apoptotic cells were defined as Annexin V-positive and PI-negative cells. Late apoptotic cells were defined as Annexin V-positive and PI-positive cells. The total number of apoptotic cells included early and late apoptotic cells (18). Each experiment was analyzed in triplicate and at least three independent experiments were performed.

Measurement of caspase- 3 and caspase- 7 activities. The activities of caspase-3 and caspase-7 were measured using the Caspase-Glo 3/7 Assay (Promega Corporation). Briefly, $\left(5 \times 10^{3}\right.$ cells per well) HCC cells were seeded into a 96-well white-walled plate and incubated under normal culture conditions for $24 \mathrm{~h}$. The cells were treated with a series of cisplatin concentrations $(0-5,000 \mathrm{ng} / \mathrm{ml})$ under normal culture condition for $72 \mathrm{~h}$. Subsequently, cells were incubated with $100 \mu \mathrm{l}$ Caspase-Glo 3/7 reagent at room temperature for $1 \mathrm{~h}$. Fluorescence signal was measured using a luminometer 
A

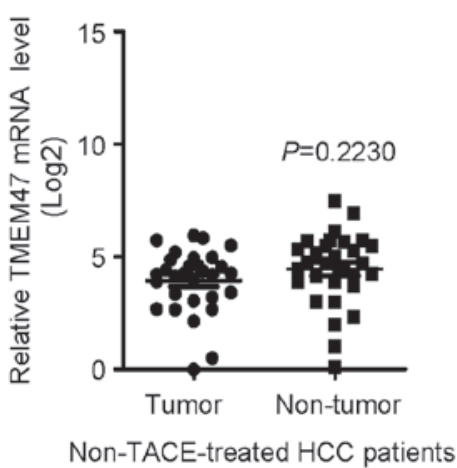

B

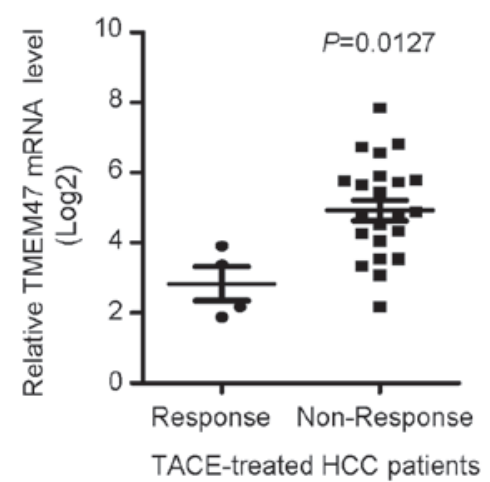

C

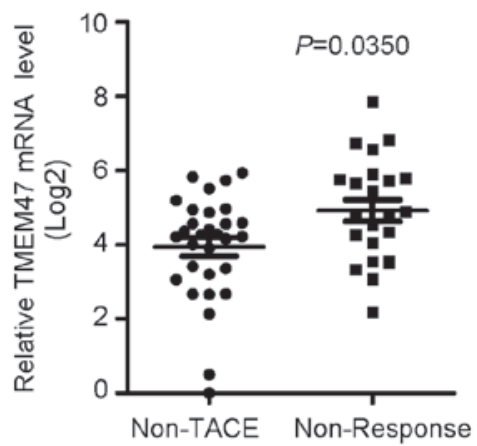

Figure 1. TMEM47 expression levels in relation to cisplatin-based TACE treatment in patients with HCC. (A) Expression levels of TMEM47 mRNA in tumor and non-tumor liver tissues of HCC patients not receiving TACE treatment. (B) Expression levels of TMEM47 mRNA in tumor tissues between HCC patients with complete response (response group) and without response (non-response group) after cisplatin TACE treatment. (C) Expression levels of TMEM47 mRNA in tumor tissues of HCC patients not receiving TACE treatment (non-TACE group) and non-response patients. TMEM47, transmembrane protein 47; TACE, transarterial chemoembolization; HCC, hepatocellular carcinoma.

(Bio-Rad Laboratories, Inc.). Each experiment was analyzed in triplicate and at least three independent experiments were performed.

$R T^{2}$ PCR array analysis. The expression of genes critical in drug metabolism was simultaneously analyzed using an $\mathrm{RT}^{2}$ Profiler PCR array (Qiagen Sciences, Inc.). Total RNA (500 ng) was extracted from HCC cells was reverse-transcribed to cDNA using the RT ${ }^{2}$ First Strand kit (Qiagen Sciences, Inc.). Each Real-Time Human Drug Metabolism PCR plate (cat. no. PAHS-002ZA; Qiagen Sciences, Inc.) contained 84 key genes associated with drug metabolism, phase I and phase II metabolizing enzymes and housekeeping genes for internal control. qPCR was performed using the SYBR-Green Master Mix (Qiagen Sciences, Inc.) on a ViiA-7 PCR machine (Thermo Fisher Scientific, Inc.). Different gene expression in HCC cells under cisplatin treatment was calculated as the fold-difference using the $2^{-\Delta \Delta \mathrm{Cq}}$ method (28) relative to the expression of HCC cells without cisplatin treatment. The mean Cq value of commonly used housekeeping genes (GAPDH and $\beta$-actin) was used as the internal control for normalization.

Animal model. A total of 24 male nude mice (aged 4-5 weeks and weighing 16-18 g) were obtained from the Laboratory Animal Unit of the University of Hong Kong. All mice were housed in individual ventilated cages in a temperature-controlled room at $\sim 25^{\circ} \mathrm{C}$ with alternating 12 -h light/dark cycles (light: 8:00 a.m.-8:00 p.m.) with ad libitum access to food and water. A xenograft ectopic liver tumor model in nude mice was established (27). HCC cells $\left(1 \times 10^{6}\right)$ were suspended in $100 \mu \mathrm{l}$ saline and subcutaneously injected into the skin on the back of the nude mice. Cisplatin (intraperitoneal injection, $5 \mathrm{mg} / \mathrm{kg} /$ week) was administered to the nude mice from the second day after subcutaneous injection of tumor cells for 3 weeks. Tumor volume was calculated as follows: Volume $\left(\mathrm{cm}^{3}\right)=1 / 2 \mathrm{x}$ length $\mathrm{x}$ width $\mathrm{x}$ width. A total of 6 nude mice were assigned to each of four experimental groups as follows: Group 1, MHCC97-control; group 2, MHCC97L-LentiTMEM47; group 3,MHCC97L/CisR-control; and group 4, MHCC97L/CisR-shTMEM47. The animal study protocol was approved by Animal (Control of Experiments) Ordinance Chapter 340, Department of Health, Hong Kong Special Administrative Region [ref.: (18-127) in DH/SHS/8/2/3 Pt. 12].

Statistical analysis. Statistical analysis was performed using GraphPad Prism version 5.01 (GraphPad Software, Inc.). Continuous variables were analyzed using the Mann-Whitney $\mathrm{U}$ test or Student's t-test. Correlation of genes was analyzed using Spearman's correlation analysis. $\mathrm{P}<0.05$ was considered to indicate a statistically significant difference.

\section{Results}

TMEM47 is positively correlated with cisplatin resistance of $H C C$. There was no significant difference in the expression levels of TMEM47 mRNA between tumor and non-tumor tissues of patients with $\mathrm{HCC}$ who did not receive TACE treatment (Fig. 1A) To investigate the clinical significance of TMEM47 in relation to the response of HCC to chemotherapy, the expression levels of TMEM47 were analyzed in HCC tissues of patients who received cisplatin-based TACE by RT-qPCR. The expression levels of TMEM47 mRNA in HCC patients without response to TACE treatment (non-response group) was significantly higher compared with that in HCC patients with complete response to TACE treatment (response group; Fig. 1B). Moreover, the expression levels of TMEM47 mRNA in the non-response group was significantly higher compared with that in HCC patients who did not receive TACE treatment (non-TACE group; Fig. 1C). Next, the expression levels of TMEM47 in the cisplatin-resistant HCC cell lines MHCC97L/CisR and MHCC97L/CisR2 and the parental HCC cell line MHCC97L were evaluated. MHCC97L/CisR2 cells exhibited a higher resistance to cisplatin compared with MHCC97L/CisR cells (18). It was observed that the TMEM47 mRNA expression levels in the two cisplatin-resistant cell lines were significantly higher compared with those in the parental MHCC97L cell line, and TMEM47 mRNA expression was the highest in MHCC97L/CisR2 cells (Fig. 2A). The expression levels of TMEM47 mRNA were not detectable in 
A

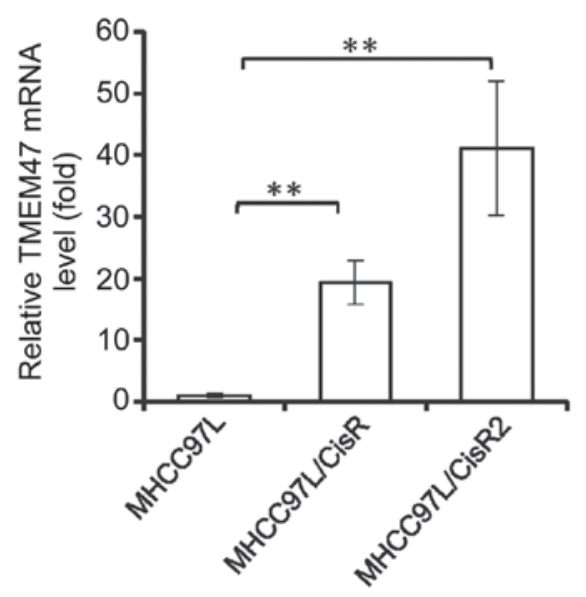

B

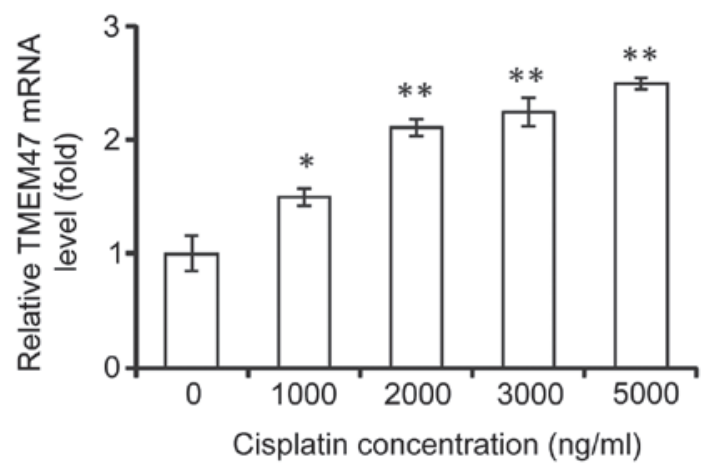

Figure 2. TMEM47 expression levels in relation to cisplatin resistance of HCC cells. (A) Expression levels of TMEM47 mRNA in parental (MHCC97L) and cisplatin-resistant (MHCC97L/CisR and MHCC97L/CisR2) HCC cell lines. (B) Expression levels of TMEM47 mRNA in MHCC97L cell line in response to cisplatin treatment. ${ }^{*} \mathrm{P}<0.05$ and ${ }^{* *} \mathrm{P}<0.01$. TMEM47, transmembrane protein 47 ; HCC, hepatocellular carcinoma.

the normal liver cell line MIHA (data not shown). In addition, when parental MHCC9L cells were exposed to increasing concentrations of cisplatin over $48 \mathrm{~h}$, the expression level of TMEM47 was upregulated and was positively associated with cisplatin concentration in a dose-dependent manner (Fig. 2B). These results indicated that TMEM47 expression responds to cisplatin treatment and is positively correlated with cisplatin resistance of $\mathrm{HCC}$.

Overexpression of TMEM47 promotes HCC resistance to cisplatin. It was next investigated whether an increase in TMEM47 expression promotes resistance of HCC to cisplatin. TMEM47-overexpressing lentiviral particles were transduced to parental MHCC97L cells to overexpress full-length TMEM47. The expression level of TMEM47 mRNA was significantly upregulated in MHCC97L-LentiTMEM47 stable cells compared with MHCC97L-control (Fig. 3A). The TMEM47 protein was also notably overexpressed in the MHCC97L-LentiTMEM47 cells (Fig. 3A). MTT assay revealed that MHCC97L-LentiTMEM47 cells exhibited a significantly increased cisplatin resistance compared with MHCC97L-control cells (Fig. 3B). The $\mathrm{IC}_{50}$ value of cisplatin for MHCC97L-control and MHCC97L-LentiTMEM47 was calculated to be 2.1 and $3.0 \mathrm{mg} / \mathrm{ml}$, respectively. Moreover, the colony-forming ability of MHCC97L-LentiTMEM47 cells under cisplatin treatment was significantly higher compared with that of MHCC97L-control cells (Fig. 3C). Furthermore, an in vivo xenograft ectopic nude mouse model demonstrated that MHCC97L-LentiTMEM47 cells exhibited a more resistant phenotype to cisplatin treatment compared with MHHC97L-control cells (Fig. 3D). Taken together, these data indicated that TMEM47 overexpression can promote cisplatin resistance of $\mathrm{HCC}$.

Targeted suppression of TMEM47 reduces cisplatin resistance of chemoresistant HCC cells. In order to investigate whether targeted inhibition of TMEM47 may be a potential therapeutic strategy for reversing cisplatin resistance of HCC, a cell line exhibiting TMEM47 suppression was established from the
MHCC97L/CisR cell line by transduction of shTMEM47 lentiviral particles. RT-PCR analysis revealed that the expression levels of TMEM47 in MHCC97L/CisR-shTMEM47 cells was significantly lower compared with that in MHCC97L/CisRcontrol cells, indicating successful suppression of TMEM47 expression (Fig. 4A). The MTT cytotoxicity assay indicated that the viability of MHCC97L/CisR-shTMEM47 cells under cisplatin treatment was significantly suppressed compared with that of MHCC97L/CisR-control cells (Fig. 4B). The $\mathrm{IC}_{50}$ value of cisplatin for MHCC97L/CisR-control and MHCC97L/CisR-shTMEM47 cells was calculated to be 15 and $8 \mathrm{mg} / \mathrm{ml}$, respectively. Moreover, the colony-forming ability of MHCC97L/CisR-shTMEM47 cells under cisplatin treatment was significantly lower compared with that of MHCC97L/CisR-control cells (Fig. 4C). In vivo experiments demonstrated that suppression of TMEM47 expression significantly enhanced the therapeutic efficacy of cisplatin in suppressing the in vivo growth rate of MHCC97L/CisR cells. This was reflected by the significantly smaller size of subcutaneous tumors formed from MHCC97L/CisR-shTMEM47 cells compared with MHCC97L/CisR-control cells after cisplatin treatment (Fig. 4D). Thus, the in vitro and in vivo results indicated a therapeutic potential of targeted inhibition of TMEM47 for overcoming cisplatin resistance of HCC.

Targeted inhibition of TMEM47 reduces the anti-apoptotic ability of cisplatin-resistant HCC cells. Anti-apoptosis is one of the major mechanisms involved in the resistance of HCC to chemotherapy (4). The present study investigated whether inhibition of TMEM47 could affect the apoptotic process of cisplatin-resistant HCC cells under cisplatin treatment. The apoptosis assay indicated that, upon increasing concentrations of cisplatin, the percentage of apoptotic MHCC97L/CisRshTMEM47 cells was significantly higher compared with that of MHCC97L/CisR-control cells (Fig. 5A). This was validated by that the significantly higher caspase- 3 and caspase- 7 activities in MHCC97L/CisR-shTMEM47 cells under cisplatin treatment compared with MHCC97L/CisR-control cells (Fig. 5B). Therefore, these results indicated that 
A

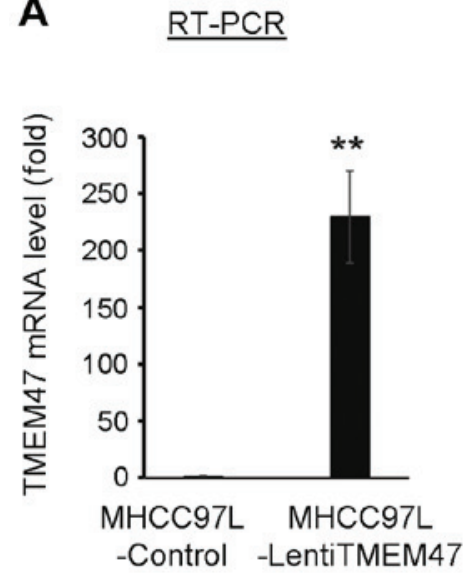

Western blot
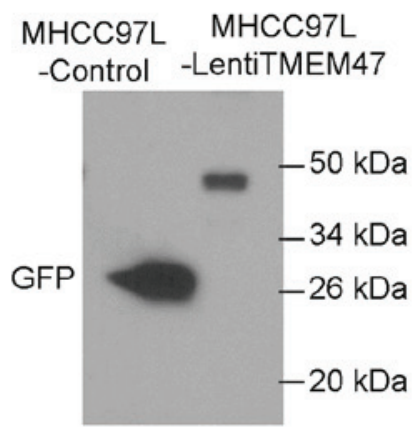

Actin $-50 \mathrm{kDa}$
B

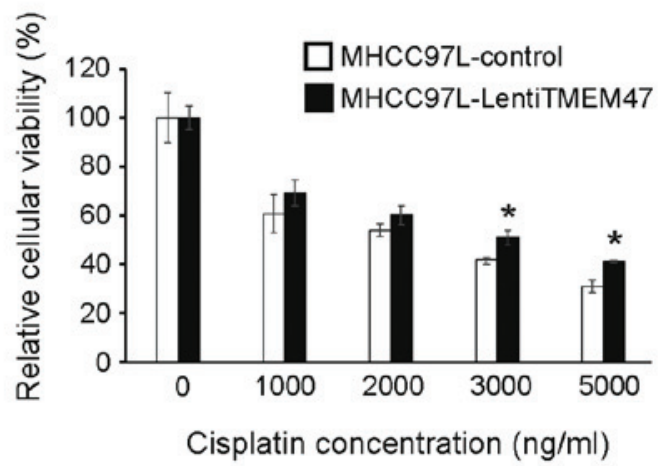

C

MHCC97LControl

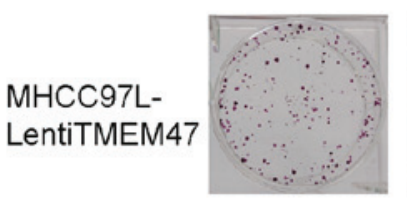

500
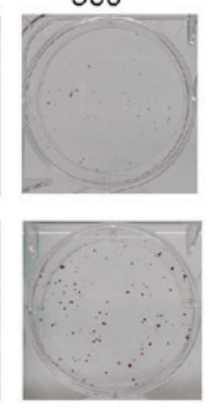

1000
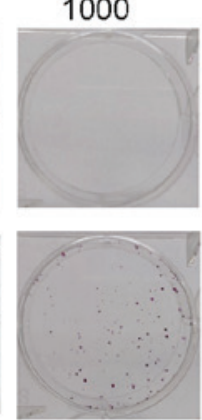

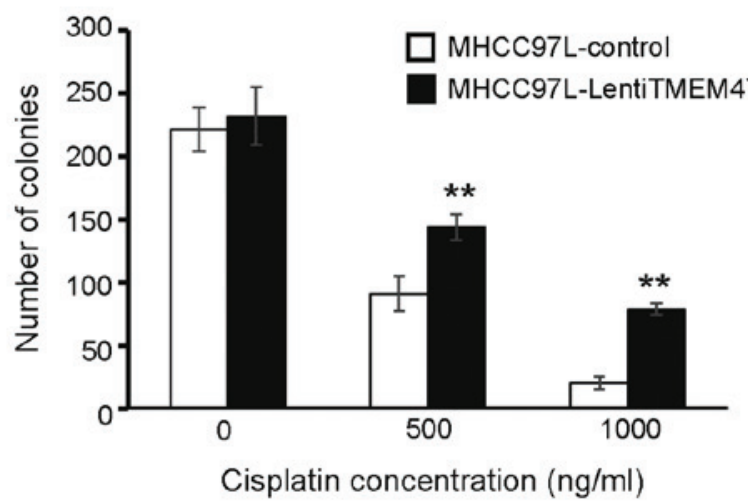

D
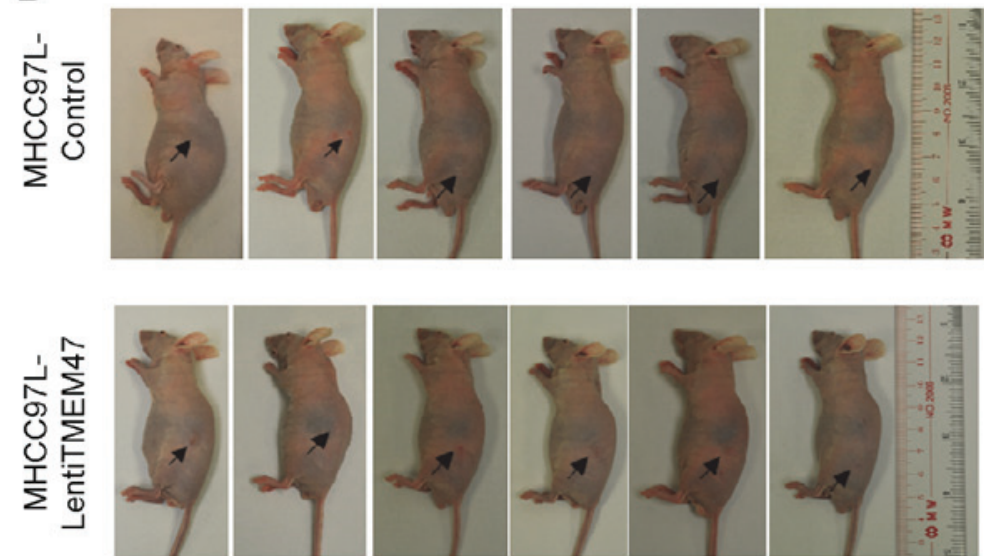

Figure 3. Overexpression of TMEM47 increases cisplatin chemoresistance of HCC. (A) Expression levels of GSTA2 mRNA and protein in MHCC97L-control and MHCC97L-lentiTMEM47 cell lines. (B) MTT assay of MHCC97L-control and MHCC97L-lenti-TMEM47 cells under cisplatin treatment. (C) Colony formation assay of MHCC97L-control and MHCC97L-lenti-TMEM47 cells under cisplatin treatment. (D) In vivo subcutaneous tumor model in nude mice injected with MHCC97L-control and MHCC97L-lentiTMEM47 cells. " $\mathrm{P}<0.05$ and ${ }^{* * *} \mathrm{P}<0.01$. TMEM47, transmembrane protein 47; HCC, hepatocellular carcinoma.

TMEM47 inhibition may reverse the anti-apoptotic ability of cisplatin-resistant HCC cells.

Targeted inhibition of TMEM47 may suppress the activation of drug metabolic genes in chemoresistant HCC cells in response to cisplatin. Increase in the expression of genes involved in drug efflux and intracellular drug metabolism is an important mechanism underlying the resistance of $\mathrm{HCC}$ to different drugs (4). The present study evaluated the effect of TMEM47 suppression on the expression levels of drug metabolic genes in cisplatin-resistant $\mathrm{HCC}$ cells using the Human Drug Metabolism RT ${ }^{2}$ profiler PCR array. It was demonstrated that, in MHCC7L/CisR-control cells, 22 genes were differentially upregulated ( $>1.5$-fold increase) under 
A

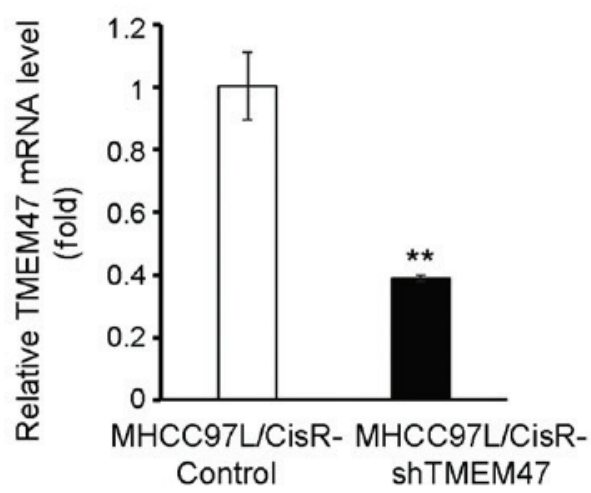

B

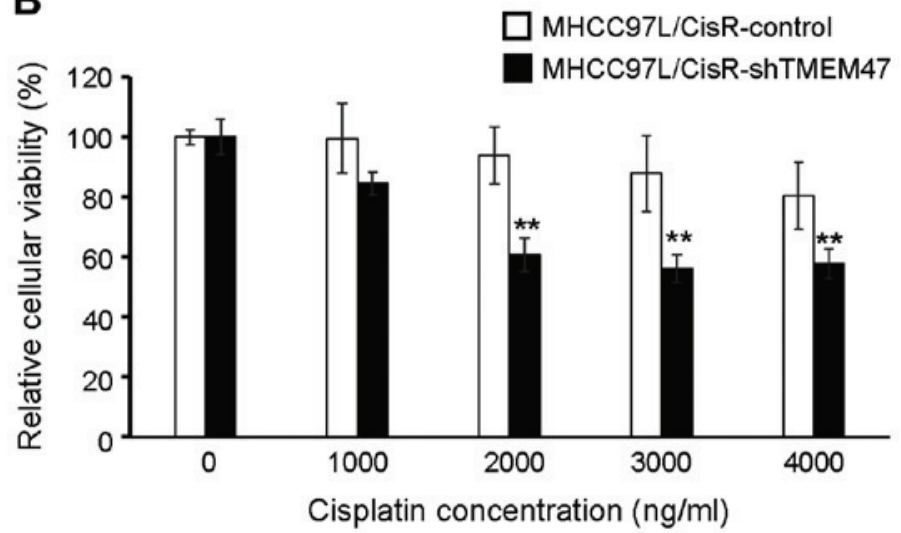

C Cisplatin concentration $(\mathrm{ng} / \mathrm{ml})$

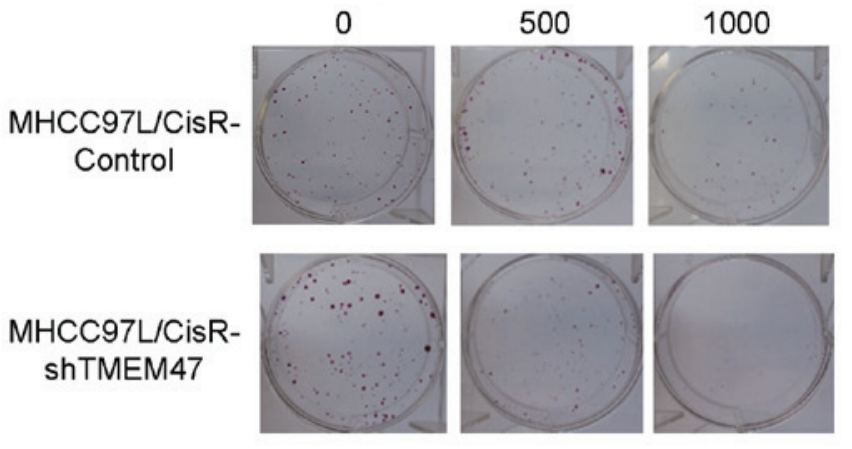

D
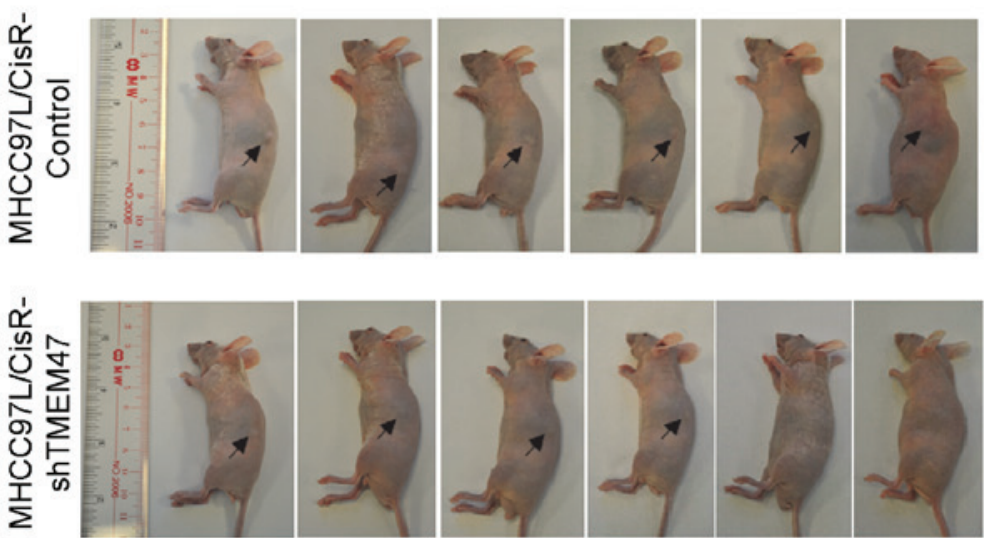

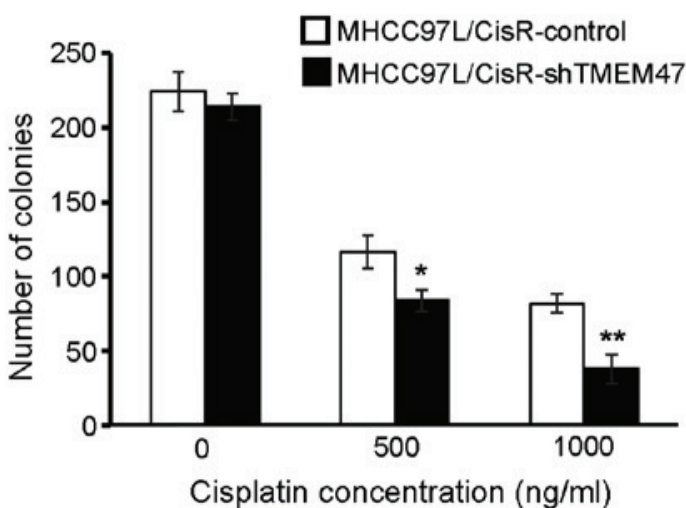

Figure 4. Suppression of TMEM47 reduces cisplatin chemoresistance of cisplatin-resistant HCC cells. (A) Reverse transcription-PCR analysis of TMEM47 mRNA MHCC97L/CisR-control and MHCC97L/CisR-shTMEM47 cell lines. (B) MTT assay of MHCC97L/CisR-control and MHCC97L/CisR-shTMEM47 cells under cisplatin treatment. (C) Colony formation assay of MHCC97L/CisR-control and MHCC97L/CisR-shTMEM47 cells under cisplatin treatment. (D) In vivo subcutaneous tumor model in nude mice injected with MHCC97L/CisR-control and MHCC97L/CisR-shTMEM47 cells. ${ }^{*}<0.05$ and ${ }^{* *} \mathrm{P}<0.01$. TMEM47, transmembrane protein 47; HCC, hepatocellular carcinoma; shTMEM47, short hairpin RNA targeting TMEM47.

cisplatin treatment (1,000 and 2,000 ng/ml; Fig. 6A). These upregulated genes included phase I metabolizing enzymes [CYP2J2, CYP11B2, cytochrome P450, family 2, subfamily E, polypeptide 1 (CYP2E1), CYP2F1, CYP2C8, CYP3A5 and CYP2C9], glutathione peroxidases (MPO, GPX5 and LPO), metallothionein (MT2A), hydrolase (FAAH), lipoxygenases (ALOX15 and APOE), decarboxylases (GAD1 and GAD2), hexokinase 2 (HK2), dehydrogenase (ADH1B), oxidoreductase (ABP1), p-glycoprotein family member (ABCC1) and other drug metabolism-related genes (GCKR and
CHST1). By contrast, 13 of these cisplatin-induced genes (MT3, CHST1, MPO, GPX5, FAAH, ALOX15, CYP11B2, CYP2E1, GAD2, CYP2F1, HK2, GAD1 and ABCC1) were not upregulated and some were even downregulated in MHCC97L/CisR-shTMEM47 cells (Fig. 6A). In addition, the number of upregulated genes in response to cisplatin in MHCC97L/CisR-shTMEM47 cells was lower compared with that in MHCC97L/CisR-control cells (15 vs. 22 genes, respectively). $\mathrm{ABCC} 1$ (also referred to as MRP1) is one of the p-glycoprotein members regulating the efflux of drugs and 
A

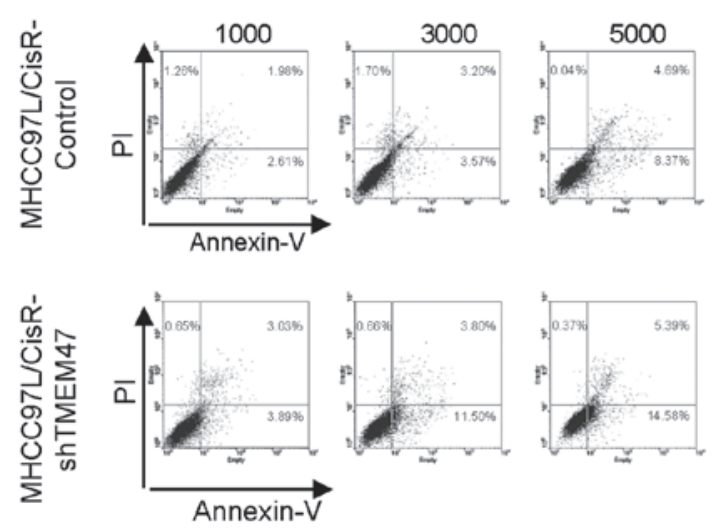

Cisplatin concentration $(\mathrm{ng} / \mathrm{ml})$

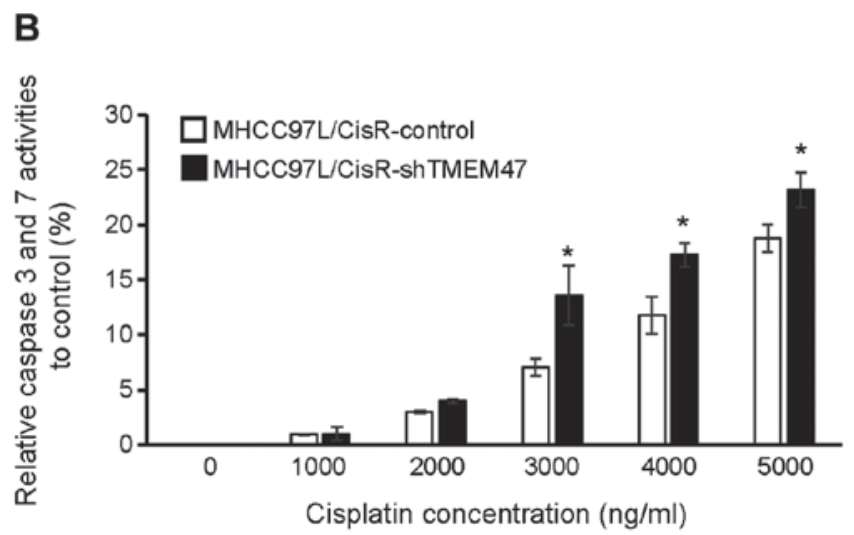

Figure 5. Suppression of TMEM47 enhances the sensitivity of cisplatin-resistant cell lines to cisplatin via increasing apoptosis and affecting the expression of drug-associated genes. (A) Apoptosis assay of MHCC97L/CisR-control and MHCC97L/CisR-shTMEM47 cells under cisplatin treatment. (B) Caspase 3/7 analysis of MHCC97L/CisR-control and MHCC97L/CisR-shTMEM47 cells under cisplatin treatment. "P<0.05 and ** P<0.01. TMEM47, transmembrane protein 47; shTMEM47, short hairpin RNA targeting TMEM47.

plays an important role in HCC resistance to cisplatin (29). It was observed that, under cisplatin treatment, ABCC1 was upregulated in MHCC97L/CisR-control cells, while its expression was suppressed in MHCC97L/CisR-shTMEM47 cells (Fig. 6A). It was further validated that the expression levels of the TMEM47 gene were significantly correlated with the $\mathrm{ABCC} 1$ expression levels in both tumor and non-tumor tissues in patients with HCC treated with TACE (Fig. 6B). These results indicated that targeted inhibition of TMEM47 may enhance the sensitivity of cisplatin-resistant HCC cells to cisplatin via suppressing cisplatin-induced activation of genes associated with drug efflux and drug metabolism.

\section{Discussion}

The prognosis of patients with HCC, particularly those with advanced disease, is poor due to the limited surgical options and progressiveness of the tumors. Although there have been significant improvements in chemotherapy and molecular-targeted therapy of patients with HCC over the past decades, chemoresistance remains a significant obstacle to treatment success $(1,4)$. The molecular mechanisms underlying $\mathrm{HCC}$ chemoresistance, which include intrinsic and acquired pathways, are complicated and multifactorial, hindering the development of effective drugs to eradicate resistant cancer cells $(4,30,31)$. To date, there is no effective strategy for overcoming HCC chemoresistance, and there is also lack of effective biomarkers for predicting response to chemotherapy or molecular-targeted therapy $(1,32)$. Therefore, identification of novel chemoresistance-associated targets is crucial not only for uncovering the molecular mechanisms implicated in HCC chemoresistance, but also for developing new effective therapeutic strategies (33). We previously established chemoresistant HCC cell lines and identified a panel of chemoresistance-associated genes, and TMEM47 was among the differentially upregulated genes identified in chemoresistant HCC cell lines (18). The role of TMEM47 in cancers is controversial, as it may either promote tumor aggressiveness or exert anti-tumor effects (20-22). To the best of our knowledge, there have been no studies investigating the role of TMEM47 in HCC and chemoresistance to date. The present study aimed to determine whether TMEM47 may be of value as a novel predictive marker and therapeutic target in HCC chemoresistance.

TACE with chemotherapeutic drugs, such as cisplatin and doxorubicin, has been widely used as the standard treatment for improving the survival of patients with intermediate- or advanced-stage HCC $(15,16)$. Although a previous study demonstrated improved survival and low mortality rates using TACE with lipiodol in 8,510 patients with unresectable 
A

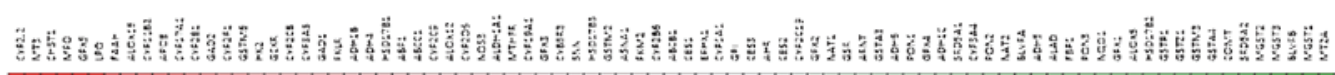

Control

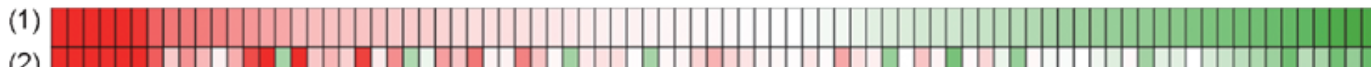

shTMEM47

(1)
(2)

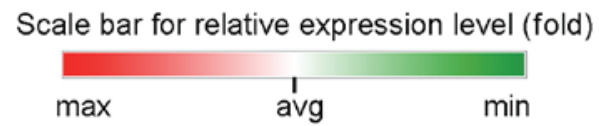

B

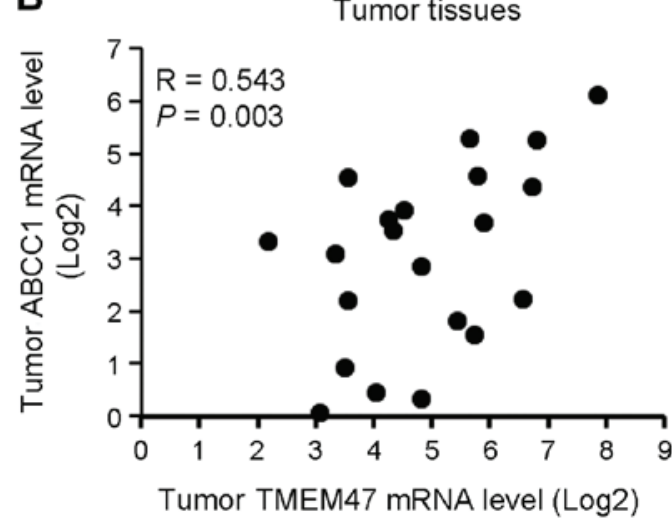

Non-Tumor tissues

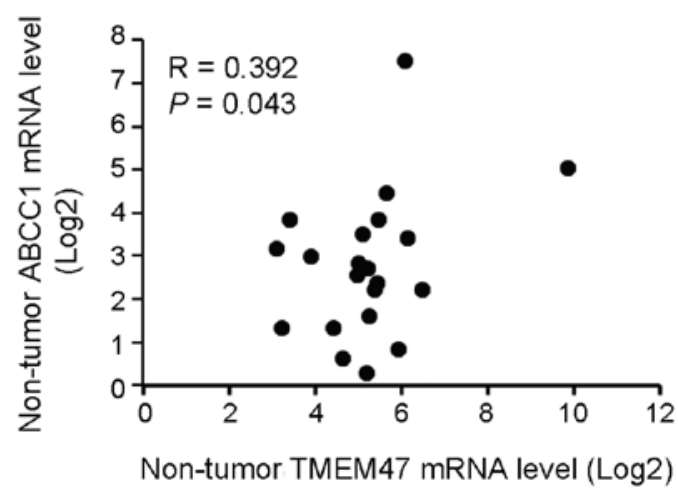

Figure 6. Targeted inhibition of TMEM47 represses the activation of cisplatin-induced drug metabolic genes. (A) RT ${ }^{2}$ PCR profiling of drug metabolic genes in MHCC97L/Cis-control and MHCC97L-shTMEM47 cell lines under cisplatin treatment. (1), $1 \mu \mathrm{g} / \mathrm{ml}$; (2) $2 \mu \mathrm{g} / \mathrm{ml}$. (B) Correlation analysis of TMEM47 and ABCC1 mRNA in tumor and non-tumor tissues from patients with HCC receiving cisplatin-based TACE treatment. TMEM47, transmembrane protein 47; shTMEM47, short hairpin RNA targeting TMEM47; HCC, hepatocellular carcinoma; TACE, transarterial chemoembolization; ABCC1, multidrug resistance-associated protein 1 .

HCC (34), the survival rate of advanced HCC following TACE treatment remains unsatisfactory (15-17), indicating a need to identify the molecular mechanisms underlying chemoresistance in HCC. The results of the present study indicated that TMEM47 expression was upregulated during cisplatin-based TACE treatment and the expression levels of the TMEM47 gene in HCC patients not responding to cisplatin-based TACE treatment were significantly higher compared with those patients with complete response. In vitro studies also indicated that cisplatin upregulated the expression of TMEM47 in a dose-dependent manner, and its expression in HCC cells was positively correlated to the degree of cisplatin resistance. Thus, these data indicated that TMEM47 may be a potential biomarker for the chemoresistance of HCC following TACE treatment. However, the sample size of TACE-treated patients in the present study was insufficient to reach a definitive conclusion, mainly due to the lack of HCC patients who were subjected to TACE prior to surgical treatment.

The function of TMEM47 in the chemoresistance of HCC was characterized via overexpression of TMEM47 in chemosensitive HCC cells. It was observed that overexpression of TMEM47 could enhance the in vitro and in vivo chemoresistance of HCC cells to cisplatin, suggesting that acquired TMEM47 expression in HCC may be a novel mechanism conferring cisplatin chemoresistance. To investigate whether TMEM47 is a potential therapeutic target for overcoming HCC chemoresistance, viral-mediated RNA interference was employed to inhibit TMEM47 expression in a cisplatin-resistant cell line and to characterize the subsequent effects on response to cisplatin. The present results demonstrated that targeted inhibition of TMEM47 expression significantly enhanced the in vitro and in vivo sensitivity of cisplatin-resistant HCC cells to cisplatin, indicating that targeted inhibition of TMEM47 may be a potential therapeutic strategy for overcoming cisplatin resistance of HCC.

Investigating the molecular mechanisms of action of TMEM47 in cisplatin resistance is crucial for the development of effective therapies for overcoming cisplatin resistance of HCC. Anti-apoptosis is one of the main mechanisms underlying the resistance of HCC to chemotherapy (4). HCC can employ diverse mechanisms to impair the drug-induced activation of apoptotic pathways, such as downregulation of p53-dependent death receptor CD95 (35), inhibition of Fas-associated death domain signaling (36), overexpression of the anti-apoptotic proteins $\mathrm{Bcl} 2$ and Bcl-xl (37) and gain-of-function mutations in P53 (38). An improved understanding of the molecular mechanisms regulating $\mathrm{HCC}$ apoptosis in the development of chemoresistance may provide valuable information for developing effective single-agent and combined therapeutic interventions. The results of the present study demonstrated that targeted inhibition of TMEM47 in cisplatin-resistant cells may enhance activation of the caspase cascade followed by intensified apoptosis under cisplatin treatment, indicating that TMEM47 may be a potential therapeutic target for overcoming anti-apoptosis-associated chemoresistance in HCC. 
Reduction of drug uptake and enhancement of drug efflux and intracellular drug metabolism are frequently observed chemoresistance-associated mechanisms in HCC that act protectively against the cytotoxic effect of drugs $(4,32)$. These acquired chemoresistant properties of HCC are attributed mainly to upregulation of multi-drug resistance genes and overexpression of phase I and phase II metabolic and detoxifying enzymes (4,32). Identification of molecular targets involved in regulating drug uptake, efflux and intracellular drug metabolism may improve our current knowledge on overcoming acquired resistance in HCC during chemotherapy. The results of the present study demonstrated that targeted inhibition of TMEM47 in cisplatin-resistant HCC cells may repress different cisplatin-induced upregulated genes involved in drug efflux and phase I and phase II metabolic enzymes, suggesting that TMEM47 may play a role in maintaining chemoresistance of $\mathrm{HCC}$, at least partly via affecting these important detoxification processes. $\mathrm{ABCC} 1$ is one of the upregulated p-glycoproteins that play a key role in cisplatin resistance in HCC (29). It was found that suppression of TMEM47 in cisplatin-resistant HCC cells could inhibit the activation of the ABCC1 gene under cisplatin treatment. Additionally, the expression levels of the TMEM47 gene were significantly associated with the ABCC1 gene expression levels in $\mathrm{HCC}$ patients receiving cisplatin-based TACE treatment. These data indicated a concurrent upregulation of TMEM47 and ABCC1 in acquired cisplatin resistance in HCC. Further experiments are required to fully characterize the effect and underlying mechanism of TMEM47 on the functions of $\mathrm{ABCC} 1$ in $\mathrm{HCC}$ chemoresistance. Whether $\mathrm{ABCC} 1$ is important for TMEM47 to confer cisplatin resistance in HCC must also by determined by inhibiting the expression of $\mathrm{ABCC} 1$ in TMEM47-overexpressing cells, followed by evaluation of cisplatin sensitivity. In addition to $\mathrm{ABCC} 1$, two differentially expressed genes in cells with TMEM47 suppression that underwent cisplatin treatment, namely CYP2E1 and HK2, were shown to be involved in cisplatin resistance in $\operatorname{HCC}(39,40)$, indicating the possible role of TMEM47 in regulating cisplatin resistance of $\mathrm{HCC}$.

Further experiments a required to support the conclusions of the present study. The present study only investigated TMEM47 mRNA levels in HCC patients with or without TACE treatment, and it would be of value to further examine the expression levels of the TMEM47 protein in patients with HCC treated with TACE by western blotting and immunohistochemistry, and to examine the prognostic potential of TMEM47 protein in predicting post-treatment response. Moreover, due to the lack of an appropriate TMEM47 antibody for the detection of the TMEM47 protein, the present study alternatively used an anti-GFP antibody for detecting the overexpression of GFP-fused TMEM47 protein in MHCC97L-LentiTMEM47 cells. Finding a suitable anti-TMEM47 antibody in the future is necessary in order to examine the expression level of the TMEM47 protein in different TMEM47-modified HCC cells. Furthermore, it would be worth investigating the roles of TMEM47 in the resistance to other drugs, such as doxorubicin, 5-fluorouracil and sorafenib, which would elucidate whether TMEM47 is cisplatin-specific or if it is a common chemoresistance-associated molecule. In addition, our previous study identified another transmembrane protein member, TMEM98, which was implicated in the chemoresistance of $\mathrm{HCC}$ via regulating the AKT and P53 pathways (18). It was observed that the expression level of TMEM47 was not correlated with TMEM98 expression in HCC patients who did or did not receive TACE treatment (data not shown), suggesting different mechanisms through which these two transmembrane proteins mediate HCC chemoresistance. Therefore, the therapeutic efficacy of combination therapy of TMEM98 and TMEM47 targeted inhibitions with conventional and molecular-targeted drugs in overcoming HCC chemoresistance must be further characterized.

In conclusion, the present study demonstrated that TMEM47 may be a potential biomarker indicating response to chemotherapy and a potential therapeutic target for overcoming HCC chemoresistance.

\section{Acknowledgements}

The authors would like to thank the Surgical Tissue Bank, (Department of Surgery, the University of Hong Kong Shenzhen Hospital) for providing the clinical samples.

\section{Funding}

The present study was supported by The National Natural Science Foundation of China (NSFC Ref. 81572945), the Theme-based Research Scheme (grant no. T12-703/19R) and General Research Fund (grant no. 17101017) of the Research Grants Council, and the Seed Funding Programme (grant nos. 201811159027, 201711159018 and 201611159111) of the University of Hong Kong.

\section{Availability of data and materials}

The datasets used and/or analyzed during the present study are available from the corresponding author upon reasonable request.

\section{Authors' contributions}

KTN and KM designed and organized the study, analyzed and interpreted the study data and wrote the manuscript. OWY, XBL, HL, JL, YFL, MHL, YYM, XQ, CXL, WQQ, HCS and MKL performed the experiments, analyzed the data and helped to draft the output and critically reviewed the manuscript; CML interpreted the data and critically reviewed the manuscript for intellectual content. All the authors have read and approved the final version of the manuscript for publication.

\section{Ethics approval and consent to participate}

All clinical samples were collected from patients after obtaining signed informed consent, and the collection of the samples by the Surgical Tissue Bank was approved by the Ethics Committee of the Institutional Review Board of the University of Hong Kong/Hospital Authority Hong Kong West Cluster (HKU/HA HKW IRB, Ref. UW 05-359 T/1022). All animal experiments were approved by Animal (Control of Experiments) Ordinance Chapter 340, the Department of Health, Hong Kong Special Administrative Region [Ref.: (18-127) in DH/SHS/8/2/3 Pt. 12]. 


\section{Patient consent for publication}

Not applicable.

\section{Competing interests}

All the authors declare that they have no competing interests.

\section{References}

1. Villanueva A: Hepatocellular carcinoma. N Engl J Med 380 1450-1462, 2019.

2. Zhu AX: Systemic therapy of advanced hepatocellular carcinoma: How hopeful should we be? Oncologist 11: 790-800, 2006.

3. Worns MA, Weinmann A, Schuchmann M and Galle PR: Systemic therapies in hepatocellular carcinoma. Dig Dis 27: $175-188,2009$.

4. Lohitesh K, Chowdhury R and Mukherjee S: Resistance a major hindrance to chemotherapy in hepatocellular carcinoma: An insight. Cancer Cell Int 18: 44, 2018.

5. Llovet JM, Ricci S, Mazzaferro V, Hilgard P, Gane E, Blanc JF, de Oliveira AC, Santoro A, Raoul JL, Forner A, et al: Sorafenib in advanced hepatocellular carcinoma. N Engl J Med 359: 378-390, 2008

6. Kudo M, Finn RS, Qin S, Han KH, Ikeda K, Piscaglia F, Baron A, Park JW, Han G, Jassem J, et al: Lenvatinib versus sorafenib in first-line treatment of patients with unresectable hepatocellular carcinoma: A randomised phase 3 non-inferiority trial. Lancet 391: 1163-1173, 2018.

7. da Motta Girardi D, Correa TS, Crosara Teixeira M and Dos Santos Fernandes G: Hepatocellular carcinoma: Review of targeted and immune therapies. J Gastrointest Cancer 49: 227-236, 2018

8. Faivre S, Rimassa L and Finn RS: Molecular therapies for HCC: Looking outside the box. J Hepatol 72: 342-352, 2020.

9. Dasari S and Tchounwou PB: Cisplatin in cancer therapy: Molecular mechanisms of action. Eur J Pharmacol 740: 364-378, 2014.

10. Ikeda K: Recent advances in medical management of hepatocellular carcinoma. Hepatol Res 49: 14-32, 2019.

11. Galluzzi L, Senovilla L, Vitale I, Michels J, Martins I, Kepp O, Castedo M and Kroemer G: Molecular mechanisms of cisplatin resistance. Oncogene 31: 1869-1883, 2012.

12. Koberle B, Tomicic MT, Usanova S and Kaina B: Cisplatin resistance: Preclinical findings and clinical implications. Biochim Biophys Acta 1806: 172-182, 2010.

13. Makovec T: Cisplatin and beyond: Molecular mechanisms of action and drug resistance development in cancer chemotherapy. Radiol Oncol 53: 148-158, 2019.

14. Pratama MY, Pascut D, Massi MN and Tiribelli C: The role of microRNA in the resistance to treatment of hepatocellular carcinoma. Ann Transl Med 7: 577, 2019.

15. Llovet JM and Bruix J: Systematic review of randomized trials for unresectable hepatocellular carcinoma: Chemoembolization improves survival. Hepatology 37: 429-442, 2003.

16. Raoul JL, Forner A, Bolondi L, Cheung TT, Kloeckner R and de Baere T: Updated use of TACE for hepatocellular carcinoma treatment: How and when to use it based on clinical evidence. Cancer Treat Rev 72: 28-36, 2019.

17. Llovet JM, Real MI, Montaña X, Planas R, Coll S, Aponte J, Ayuso C, Sala M, Muchart J, Solá R, et al: Arterial embolisation or chemoembolisation versus symptomatic treatment in patients with unresectable hepatocellular carcinoma: A randomised controlled trial. Lancet 359: 1734-1739, 2002.

18. Ng KT, Lo CM, Guo DY, Qi X, Li CX, Geng W, Liu XB, Ling CC, Ma YY, Yeung WH, et al: Identification of transmembrane protein 98 as a novel chemoresistance-conferring gene in hepatocellular carcinoma. Mol Cancer Ther 13: 1285-1297, 2014.

19. Dong Y and Simske JS: Vertebrate Claudin/PMP22/EMP22/ MP20 family protein TMEM47 regulates epithelial cell junction maturation and morphogenesis. Dev Dyn 245: 653-666, 2016.

20. Burnett RM, Craven KE, Krishnamurthy P, Goswami CP, Badve S, Crooks P, Mathews WP, Bhat-Nakshatri P and Nakshatri $\mathrm{H}$ : Organ-specific adaptive signaling pathway activation in metastatic breast cancer cells. Oncotarget 6: 12682-12696, 2015.
21. Cheung IY, Feng Y, Danis K, Shukla N, Meyers P, Ladanyi M and Cheung NK: Novel markers of subclinical disease for Ewing family tumors from gene expression profiling. Clin Cancer Res 13: 6978-6983, 2007.

22. Mithani SK, Smith IM and Califano JA: Use of integrative epigenetic and cytogenetic analyses to identify novel tumor-suppressor genes in malignant melanoma. Melanoma Res 21: 298-307, 2011.

23. Poon RT, Ngan H, Lo CM, Liu CL, Fan ST and Wong J: Transarterial chemoembolization for inoperable hepatocellular carcinoma and postresection intrahepatic recurrence. J Surg Oncol 73: 109-114, 2000

24. Li Y, Tang ZY, Ye SL, Liu YK, Chen J, Xue Q, Chen J, Gao DM and Bao WH: Establishment of cell clones with different metastatic potential from the metastatic hepatocellular carcinoma cel line MHCC97. World J Gastroenterol 7: 630-636, 2001.

25. Ng KT, Xu A, Cheng Q, Guo DY, Lim ZX, Sun CK, Fung JH, Poon RT, Fan ST, Lo CM, et al: Clinical relevance and therapeutic potential of angiopoietin-like protein 4 in hepatocellular carcinoma. Mol Cancer 13: 196, 2014

26. Ng KT, Qi X, Kong KL, Cheung BY, Lo CM, Poon RT, Fan ST and Man K: Overexpression of matrix metalloproteinase-12 (MMP-12) correlates with poor prognosis of hepatocellular carcinoma. Eur J Cancer 47: 2299-305, 2011.

27. Ng KT, Lee TK, Cheng Q, Wo JY, Sun CK, Guo DY, Lim ZX, Lo CM, Poon RT, Fan ST, et al: Suppression of tumorigenesis and metastasis of hepatocellular carcinoma by shRNA interference targeting on homeoprotein Six1. Int J Cancer 127: 859-872, 2010.

28. Livak KJ and Schmittgen TD: Analysis of relative gene expression data using real-time quantitative PCR and the 2(-Delta Delta C(T)) method. Methods 25: 402-408, 2001.

29. Liu XY, Liu SP, Jiang J, Zhang X and Zhang T: Inhibition of the JNK signaling pathway increases sensitivity of hepatocellular carcinoma cells to cisplatin by down-regulating expression of P-glycoprotein. Eur Rev Med Pharmacol Sci 20: 1098-1108, 2016.

30. Yau T, Chan P, Epstein R and Poon RT: Management of advanced hepatocellular carcinoma in the era of targeted therapy. Liver Int 29: 10-17, 2009.

31. Wysocki PJ: Targeted therapy of hepatocellular cancer. Expert Opin Investig Drugs 19: 265-274, 2010.

32. Duan B, Huang C, Bai J, Zhang YL, Wang X, Yang J and Li J: Multidrug resistance in hepatocellular carcinoma. In: Hepatocellular Carcinoma. Tirnitz-Parker JEE (ed). Codon Publications, Brisbane, AU, 2019.

33. Forner A, Llovet JM and Bruix J: Hepatocellular carcinoma. Lancet 379: 1245-1245, 2012.

34. Takayasu K, Arii S, Ikai I, Omata M, Okita K, Ichida T, Matsuyama Y, Nakanuma Y, Kojiro M, Makuuchi M, et al: Prospective cohort study of transarterial chemoembolization for unresectable hepatocellular carcinoma in 8510 patients. Gastroenterology 131: 461-469, 2006.

35. Volkmann M, Schiff JH, Hajjar Y, Otto G, Stilgenbauer F, Fiehn W, Galle PR and Hofmann WJ: Loss of CD95 expression is linked to most but not all p53 mutants in European hepatocellular carcinoma. J Mol Med (Berl) 79: 594-600, 2001

36. Schattenberg JM, Galle PR and Schuchmann M: Apoptosis in liver disease. Liver Int 26: 904-911, 2006.

37. Chun $\mathrm{E}$ and Lee KY: Bcl-2 and Bcl-xL are important for the induction of paclitaxel resistance in human hepatocellular carcinoma cells. Biochem Biophys Res Commun 315: 771-779, 2004.

38. Chan KT and Lung ML: Mutant p53 expression enhances drug resistance in a hepatocellular carcinoma cell line. Cancer Chemother Pharmacol 53: 519-526, 2004.

39. Mondal J and Khuda-Bukhsh AR: Cisplatin and farnesol co-encapsulated PLGA nano-particles demonstrate enhanced anti-cancer potential against hepatocellular carcinoma cells in vitro. Mol Biol Rep 47: 3615-3628, 2020.

40. Cassim S, Raymond VA, Dehbidi-Assadzadeh L, Lapierre P and Bilodeau M: Metabolic reprogramming enables hepatocarcinoma cells to efficiently adapt and survive to a nutrient-restricted microenvironment. Cell Cycle 17: 903-916, 2018.

This work is licensed under a Creative Commons Attribution-NonCommercial-NoDerivatives 4.0 International (CC BY-NC-ND 4.0) License. 